\title{
Age dependence of pulmonary artery blood flow measured by 4D flow cardiovascular magnetic resonance: results of a population-based study
}

\author{
Thomas Wehrum ${ }^{1 *}$, Paul Hagenlocher', Thomas Lodemann', Werner Vach², lulius Dragonu ${ }^{3}$, Anja Hennemuth', \\ Constantin von zur Mühlen ${ }^{5}$, Judith Stuplich ${ }^{5}$, Ba Thanh Truc Ngo ${ }^{5}$ and Andreas Harloff ${ }^{1}$
}

\begin{abstract}
Background: It was our aim to systematically analyze pulmonary artery blood flow within different age-groups in the general population using 4D flow cardiovascular magnetic resonance (CMR) in order to provide a context for interpreting results of future studies (e.g., in pulmonary hypertension) using this technique.

Methods: An age-stratified sample $(n=126)$ of the population of the city of Freiburg, Germany, underwent ECG-triggered and navigator-gated 4D flow CMR at 3 T of the pulmonary arteries and the thoracic aorta. Analysis planes were placed in the main, left, and right pulmonary artery using dedicated software. Study participants were divided into three groups (1:20-39; 2:40-59; and 3:60-80 years of age). Subsequently, pulmonary blood flow was visualized, quantified and compared between groups.

Results: Time-to-peak of systolic antegrade flow was shorter, peak and average velocities and flow volumes were lower in older subjects. At the end of systole, retrograde flow in the main pulmonary artery was observed in all but one subject. Subsequently, a second antegrade flow peak occurred in diastole which was lower in older subjects. Age was an independent predictor of hemodynamic change after adjustment for cardiovascular risk factors and body-mass-index. During systole, abnormal vortices occurred in the main pulmonary artery in four male subjects.

Conclusions: Comprehensive analysis of pulmonary blood flow was feasible in all subjects. We were able to detect an independent effect of ageing on pulmonary hemodynamics reflecting increased vessel stiffness and reduced pulmonary circulation. Findings of this study may be helpful for discriminating physiological from pathological flow in patients with pulmonary diseases in the future.
\end{abstract}

Keywords: Population, 4D flow CMR, Pulmonary arteries, Pulmonary blood flow

\section{Background}

Pulmonary artery blood flow is strongly dependent on adequate cardiac function and absence of systemic vascular disease and pathological conditions of the lungs. Hence, many pathologic conditions may lead to altered pulmonary hemodynamics and elevation of pulmonary arterial pressure [1]. Pulmonary hypertension (PH, i.e., the elevation of mean pulmonary arterial pressure $\geq 25 \mathrm{mmHg}$ [2])

\footnotetext{
* Correspondence: thomas.wehrum@uniklinik-freiburg.de

${ }^{1}$ Department of Neurology, University Medical Center Freiburg, Breisacher

Straße 64, 79106 Freiburg, Germany

Full list of author information is available at the end of the article
}

is a serious condition which requires accurate assessment of intrapulmonary hemodynamics and pressure to identify patients, who will benefit from treatment. Currently, the diagnostic gold-standard for intra-pulmonary pressure evaluation is right-heart catheterization, which is an invasive procedure. Hence, transthoracic echocardiography (TTE) is used as a non-invasive screening tool by assessing cardiac function and estimating pulmonary arterial pressure non-invasively [3]. Unfortunately, specificity of TTE for the identification of patients with $\mathrm{PH}$ is limited and [3], therefore, other non-invasive diagnostic tools are under investigation. One promising technique is $4 \mathrm{D}$ flow 
cardiovascular magnetic resonance (CMR), which allows time-resolved and three-dimensional assessment of hemodynamic parameters in vivo [4] and has already been applied to the pulmonary arteries: $\mathrm{PH}$ was reported to be associated with a decrease of flow parameters such as peak systolic velocity, peak flow, stroke volume [5], the increase of pulmonary artery (PA) diameter [6] and stiffness [7], early onset of retrograde flow in the main pulmonary artery (MPA) [8], and the occurrence of abnormal vortices $[9,10]$. These findings were made by examining $\mathrm{PH}$ patients and healthy volunteers [5, 7-9]. Normal pulmonary flow characteristics have previously been described using 2D phase-contrast CMR and echocardiography and have been compared with right heart catheterization indicating a higher agreement of CMR than of echocardiography regarding pulmonary flow and pressure estimation [11]. However, previous studies were limited in sample size [5, 7-9, 11]. Furthermore, 2D flow CMR is of limited use in a clinical setting because it does not allow retrospective selection of regions of interest inside a 3D data volume to perform post-hoc quantification of blood flow parameters. Using 4D flow CMR, all measurements are performed within one dataset with no need for several 2D acquisitions with inherent differences in heartrate [12]. These advantages may allow a more comprehensive study of hemodynamic change during the course of $\mathrm{PH}$. However, reference values derived from the general population are needed to interpret results of future interventional studies.

\section{Methods}

\section{Study population}

We performed a cross-sectional observational study of the population of the city of Freiburg, Germany. Our cohort was established on the basis of data obtained from the local residents' registration office. We included at least 20 subjects per decade ( 10 females and 10 males) within the age of 20-80 years. Starting in October 2012, 3500 age-stratified and randomly selected residents of Freiburg were contacted by mail, asked to participate in our study, and provided with details on how to contact the study team. 308 subjects responded to our mail and were contacted by phone on the basis of first-come, first-served. 147 had to be excluded because of reporting CMR contraindications during the telephone interview, too many participants in this group of age, or because no suitable date could be realized. 161 subjects were finally scheduled for study CMR. In 23 subjects the CMR protocol was not completed for technical reasons, 11 did not appear on the appointed day, 11 aborted CMR examination early because of claustrophobia, and 5 were not suited for CMR due to contraindications that were not evident at the preceding telephone interview. Because of insufficient response within the group of 20-29 year old males, the study was advertised on the University Hospital Freiburg intranet and men within this age-interval $(n=922)$ were invited to participate. The first 16 subjects who contacted the study team by email were consecutively included. One person had to be excluded for technical difficulties during CMR and one subject did not arrive on the appointed day. Finally, datasets of 126 subjects were available for analysis.

Cardiovascular risk factors and demographics were determined by interview on site and blood pressure was measured at the left upper arm in a supine position after 5 min rest before and after CMR examination. Heart rate was documented every 5 min during CMR blood flow measurements. All participants underwent additional transthoracic echocardiography (TTE) using a Toshiba Artida system (4.8-2 MHz PST-30BT transducer; Toshiba Medical Systems Corporation, Tokyo, Japan) based on the recommendations and standards of the American Society of Echocardiography [13] on the same day (=median) as CMR. The study was approved by the ethics committee of the University of Freiburg and written informed consent was obtained from all participants.

\section{D flow CMR}

All CMR examinations were conducted on a routine 3Tesla MR system (TIM Trio, Siemens Healthcare AG, Erlangen, Germany), using a standard 12-element body coil. 4D flow CMR was used to obtain time-resolved and threedimensional blood flow parameters of the pulmonary arteries and the thoracic aorta. All experiments used prospective ECG-gating and navigator-gating to allow free breathing [14]. Parameters of 4D flow CMR were: echo time/repetition time $(\mathrm{TE} / \mathrm{TR})=2.6 / 5.1 \mathrm{~ms}$, flip angle $=7^{\circ}$, temporal resolution $=20.4 \mathrm{~ms}$, matrix size $=320 \times 240 \times 58$, bandwidth $=450 \mathrm{~Hz} /$ pixel, spatial resolution $=2.1 \times 2.1 \times 2.5 \mathrm{~mm}$, velocity sensitivity along all three directions $=150 \mathrm{~cm} / \mathrm{s}$, and parallel imaging (PEAK-GRAPPA) along the phase encoding direction (y) with an acceleration factor of $R=5$ (20 reference lines).

\section{Data analysis}

4D flow CMR datasets were analyzed using MEVISFlow software (Fraunhofer MEVIS, Bremen, Germany) [15]. After corrections for eddy-currents and phase-wraps, the vessel was segmented and three analysis planes were manually positioned perpendicular to the vessel lumen in the main pulmonary artery (MPA), the left pulmonary artery (LPA), and the right pulmonary artery (RPA). All planes were located with a distance of $1 \mathrm{~cm}$ from the center of the bifurcation of the PA in order to avoid interference by local flow turbulences in this area. For the comparison of pulmonary and aortic blood flow a fourth analysis plane was positioned in the ascending aorta (AAo) at the level of the lower edge of the left pulmonary artery (see Fig. 1). For each analysis plane, a lumen contour surrounding the 

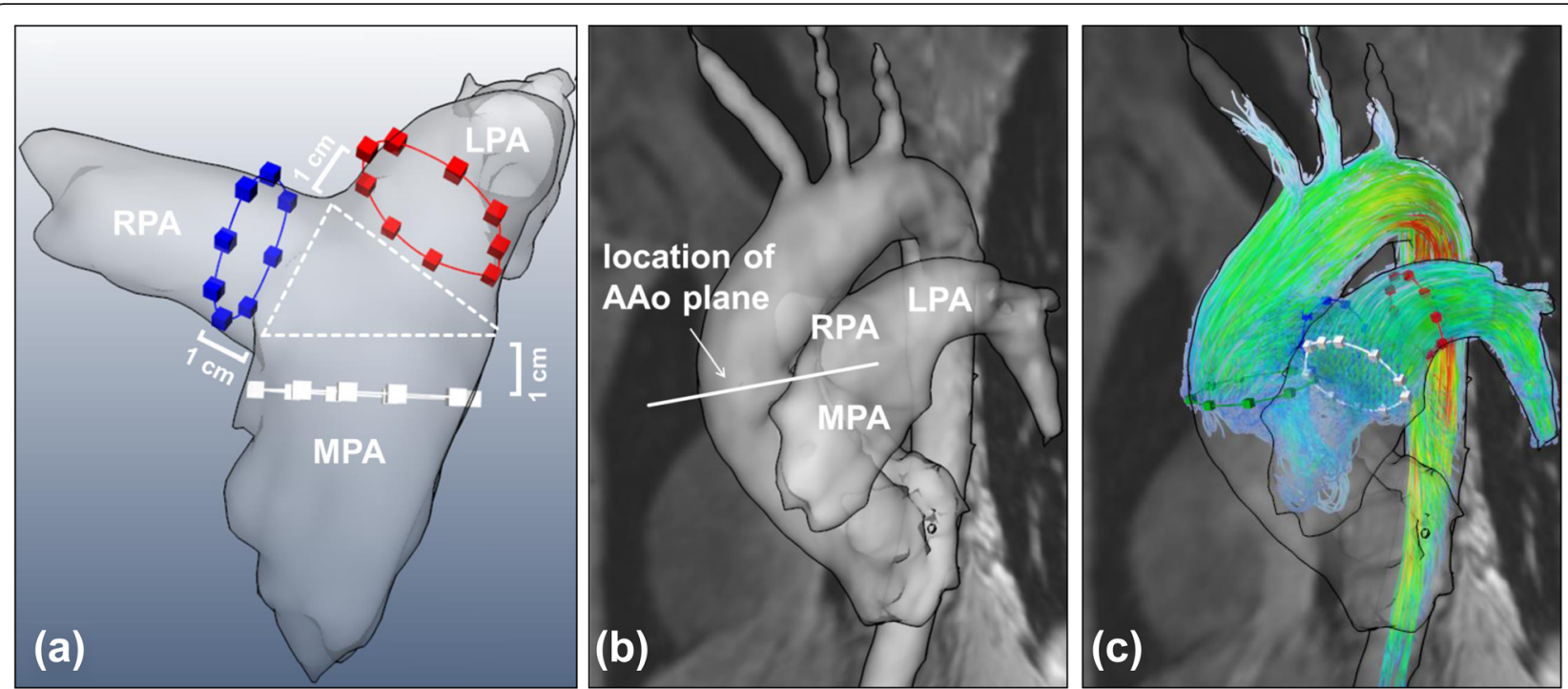

Fig. 1 Positioning of analysis planes. a Analysis planes in the MPA, LPA, and RPA were positioned with a distance of $1 \mathrm{~cm}$ distal to the MPA bifurcation. $\mathbf{b}$ The AAo plane was located in a standardized fashion on a level with the lower edge of the RPA. $\mathbf{c}$ 4D flow quantification was performed in all four analysis planes

lumen was defined manually while the contour was adapted automatically to all time points of the cardiac cycle [15].

\section{Parameters of pulmonary blood flow}

The following parameters were evaluated because they were either regarded as parameters of vascular function or have been used earlier as potential surrogates for elevated pulmonary arterial pressure $[5,6,9,10]: 1)$ minimum $\left(\mathrm{CSA}_{\min }\right)$, maximum $\left(\mathrm{CSA}_{\max }\right)$, average $\left(\mathrm{CSA}_{\text {avg }}\right)$ and change $\left(\mathrm{CSA}_{\text {change }}\right)$ of cross-sectional-area of the lumen and the MPA/AAo ratio $\left(\mathrm{CSA}_{\text {ratio }}\right)$ as calculated from $\mathrm{CSA}_{\text {min }}$, 2) time to peak systolic $\left(\mathrm{TTP}_{\text {sys }}\right)$ and peak diastolic $\left(\mathrm{TTP}_{\text {dia }}\right)$ antegrade flow and time to peak retrograde flow $\left(\mathrm{TTP}_{\text {ret }}\right)$ as measured from the beginning of the acquisition (i.e., the QRS), 3) parameters of planar blood-flow (i.e., peak $\left(\mathrm{V}_{\text {max }}\right)$ and average $\left(\mathrm{V}_{\text {avg }}\right)$ velocities in any direction, peak systolic $\left(\mathrm{Q}_{\max \text {-sys }}\right)$ and diastolic $\left(\mathrm{Q}_{\max -d i a}\right)$ flow, mean flow per cardiac cycle $\left(\mathrm{Q}_{\text {mean }}\right)$, fraction of retrograde flow (RF), and cardiac output (CO). Additionally, flow was visualized in the MPA plane and analyzed regarding the appearance of vortices (i.e., flow which is mostly rotating on an axis line) and the extent of retrograde flow, which were both associated with increased pulmonary pressure in earlier studies [8-10]. To test correctness of 4D flow CMR measures, conservation of mass was tested by measuring mean flow per cardiac cycle in the MPA $\left(\mathrm{Q}_{\mathrm{MPA}}\right)$, LPA $\left(\mathrm{Q}_{\mathrm{LPA}}\right), \mathrm{RPA}\left(\mathrm{Q}_{\mathrm{RPA}}\right)$, and the AAo $\left(\mathrm{Q}_{\mathrm{AAo}}\right)$. Provided that $4 \mathrm{D}$ flow $\mathrm{CMR}$ measurements are accurate, blood flow was expected to be the same during one cardiac cycle at the right and the left outflow tract of the heart: $\mathrm{Q}_{M P A}=\mathrm{Q}_{\mathrm{LPA}}+\mathrm{Q}_{\mathrm{RPA}}=\mathrm{Q}_{\mathrm{AAo}}$.

\section{Statistical analysis}

Data are presented as mean ( \pm standard deviations) or median (interquartile range) for continuous, absolute and relative frequencies for categorical variables. Regression analysis was performed to quantify correlations of flow between various blood vessels. Furthermore, patients were categorized according to their age into three groups which were used for comparisons of blood flow: group 1 (20-39), group 2 (40-59), and group 3 (60-80 years of age). Departures from normality were detected with the Shapiro-Wilk statistic. Homogeneity of variance was assessed using Levene's test. Differences between patient groups were evaluated using Fisher's exact test and one-way ANOVA with Tukey's HSD post-hoc test, respectively. Furthermore, trends between groups were tested using linear regression and correlations between age and hemodynamic parameters were assessed after adjustment for cardiovascular risk factors and body-mass-index. All tests were two-sided with 0.05 as the level of statistical significance. Statistical analyses were performed using IBM-SPSS Statistics version 19.0.1.

\section{Results}

\section{Patient characteristics}

Cardiovascular risk factors, patient demographics and results from TTE are presented in Table 1. All participants were caucasian. Hypertension and hypercholesterolemia occurred more often in older subjects (group $3>2>1$ ) while no differences between groups were observed regarding the remaining risk factors. Only few subjects had diabetes $(n=2)$, prior stroke $(n=2)$, coronary-arterydisease $(n=2)$, and none of the subjects suffered from peripheral vascular disease. In the onsite interview none of 
Table 1 Characteristics of study participants

\begin{tabular}{|c|c|c|c|c|}
\hline Characteristics of patients & $\begin{array}{l}\text { Group } 1 \\
(20-39 y .) \\
n=43\end{array}$ & $\begin{array}{l}\text { Group } 2 \\
(40-59 y .) \\
n=44\end{array}$ & $\begin{array}{l}\text { Group } 3 \\
(60-80 y .) \\
n=39\end{array}$ & $p$-value \\
\hline Age, years $( \pm S D)$ & $30.07( \pm 5.4)$ & $50.43( \pm 5.5)$ & $68.95( \pm 5.2)$ & $<0.001^{*}$ \\
\hline Female, $n(\%)$ & $19(44.2)$ & $24(54.6)$ & $21(53.9)$ & 0.564 \\
\hline Hypertension, $n(\%)$ & $1(2.3)$ & $7(16.3)$ & $13(33.3)$ & $<0.001^{*}$ \\
\hline Hypercholesterolemia, $n(\%)$ & $1(2.3)$ & $9(20.5)$ & $11(28.2)$ & $0.005^{*}$ \\
\hline Diabetes, $n(\%)$ & $0(0.0)$ & $1(2.3)$ & $1(2.6)$ & 0.587 \\
\hline Smoker, $n(\%)$ & $11(25.6)$ & $6(13.6)$ & $5(12.8)$ & 0.223 \\
\hline BMI, $1( \pm S D)$ & $24.02( \pm 4.0)$ & $26.03( \pm 4.4)$ & $24.31( \pm 3.8)$ & 0.051 \\
\hline Prior stroke, $n(\%)$ & $0(0.0)$ & $2(4.6)$ & $0(0.0)$ & 0.151 \\
\hline Coronary heart disease, $n(\%)$ & $0(0.0)$ & $0(0.0)$ & $2(5.1)$ & 0.104 \\
\hline Peripheral arterial disease, $n(\%)$ & $0(0.0)$ & $0(0.0)$ & $0(0.0)$ & - \\
\hline Mean systolic $\mathrm{BP}, \mathrm{mmHg}( \pm \mathrm{SD})$ & $120.63( \pm 11.1)$ & $126( \pm 15.7)$ & $133.78( \pm 19.2)$ & $<0.001^{*}$ \\
\hline Mean diastolic $\mathrm{BP}, \mathrm{mmHg}( \pm \mathrm{SD})$ & $76.53( \pm 7.0)$ & $81.88( \pm 8.3)$ & $81.09( \pm 11.4)$ & $0.014^{*}$ \\
\hline Heart rate, bpm $( \pm S D)$ & $67.16( \pm 7.9)$ & $65.55( \pm 7.7)$ & $66.1( \pm 9.1)$ & 0.655 \\
\hline Ejection fraction, $\%( \pm S D)$ & $56.6( \pm 20.7)$ & $57.6( \pm 19.2)$ & $53.6( \pm 22.5)$ & 0.669 \\
\hline PI grade I, $n(\%)$ & $3(7.0)$ & $1(2.3)$ & $1(2.6)$ & 0.246 \\
\hline PI grade II- IV, $n(\%)$ & $0(0.0)$ & $0(0.0)$ & $0(0.0)$ & - \\
\hline $\mathrm{RVSP}, \mathrm{mmHg}( \pm \mathrm{SD})$ & $15.82( \pm 2.4)$ & $18.22( \pm 4.8)$ & $22.05( \pm 5.7)$ & $<0.001^{*}$ \\
\hline TAPSE, $m m( \pm S D)$ & $22.93( \pm 4.1)$ & $22.56( \pm 3.3)$ & $23.06( \pm 3.7)$ & 0.906 \\
\hline $\mathrm{TDI}, \mathrm{cm} / \mathrm{s}(\%)$ & $15.46( \pm 2.9)$ & $15.53( \pm 1.8)$ & $15.82( \pm 1.8)$ & 0.592 \\
\hline
\end{tabular}

Demographics, cardiovascular risk factors and results from transthoracic echocardiography of study participants. SD standard deviation, $B P$ blood pressure, $P I$ pulmonary valve insufficiency, RVSP right ventricular systolic pressure, TAPSE tricuspid annular plane systolic excursion, TDI systolic velocity of the right ventricular lateral annulus, *statistically significant

the subjects reported a history of relevant lung disease (i.e., asthma, chronic obstructive pulmonary disease, interstitial lung disease, pulmonary embolism, tumor, pleural disease or acute infection of the respiratory tract) at the time of examination. In 54 (42.56\%) patients systolic PA pressure could be quantified from tricuspid regurgitation Doppler. Right ventricular systolic pressure was higher in older patients (group $3>2>1$ ) but still within the reference range according to the ASE recommendations [13] and none of the patients had signs of PH. Echocardiographic findings were within the reference range in all but two individuals, with one having a mildly abnormal leftventricular function ( $\mathrm{EF}=45 \%)$ and one having an enlarged left atrium (LA diameter $=49 \mathrm{~mm}$ ).

\section{Conservation of mass during 4D flow quantification}

Mean stroke volume of all subjects during one cardiac cycle was $75.5 \pm 17.2 \mathrm{ml}$ in the MPA, a total of $74.1 \pm 17.5 \mathrm{ml}$ in the LPA $(34.0 \pm 8.8 \mathrm{ml})$ plus RPA $(40.1 \pm 9.6 \mathrm{ml})$, and 74.3 $\pm 18.2 \mathrm{ml}$ in the ascending aorta $(\mathrm{F}(2375)=0.24, p=0.788)$. Correlations were high between flow values in the MPA and RPA + LPA $(r=0.92 ; p<0.001)$, the MPA and the AAo $(r=0.88 ; p<0.001)$, and the RPA + LPA and the AAo $(r=0.91 ; p<0.001)$.

\section{Age related change of flow}

The mean cross-sectional area (CSA) of the pulmonary arteries and the ascending aorta is given in Table 2. We observed a trend that the CSA of the MPA was smaller in older subjects, while the CSA of the AAo, LPA and RPA was higher in older participants resulting in a proportionally higher $\mathrm{CSA}_{\text {ratio }}$ in younger subjects (group $1>2>3$; see Fig. 2). The $\mathrm{CSA}_{\text {change }}$ decreased with age in the MPA and AAo as a marker of increased stiffness.

The flow profile in the MPA, LPA, and RPA compromised three phases: 1) systolic antegrade flow, 2) endsystolic and early-diastolic retrograde flow (in all but one patient without any detectable retrograde flow), and 3) diastolic antegrade flow. The distribution of flow in relation to the cardiac cycle is illustrated in Fig. 3 and the position of landmarks (time-to-peak of antegrade systolic, retrograde early-diastolic, and antegrade diastolic flow) is given in Table 3. Peak systolic flow in the MPA occurred later in the cardiac cycle in younger patients (group $1>2+3$ ) while no difference was observed regarding the time point of peak retrograde flow and peak antegrade diastolic flow.

Systolic peak flow values $\left(\mathrm{Q}_{\max \text {-sys }}\right)$ and corresponding velocities $\left(\mathrm{V}_{\max }\right.$ and $\mathrm{V}_{\text {avg }}$ ) were higher in younger patients (group $1>2>3$ ) (see Fig. 3 and Table 4 ) in the left 
Table 2 Pulmonary and aortic cross-sectional area

\begin{tabular}{|c|c|c|c|c|c|}
\hline & $\begin{array}{l}\text { Group } 1 \\
(20-39 y .) \\
n=43\end{array}$ & $\begin{array}{l}\text { Group } 2 \\
(40-59 y .) \\
n=44\end{array}$ & $\begin{array}{l}\text { Group } 3 \\
(60-80 y .) \\
n=39\end{array}$ & $F(2,375)$ & $p$-value \\
\hline \multicolumn{6}{|c|}{$\overline{\mathrm{CSA}_{\max }, \mathrm{Cm}^{2}( \pm \mathrm{SD})}$} \\
\hline MPA & $6.53 \pm 1.34$ & $6.11 \pm 1.21$ & $5.78 \pm 1.17$ & 7.39 & $0.008^{*}$ \\
\hline LPA & $3.31 \pm 0.65$ & $3.55 \pm 1.23$ & $3.69 \pm 0.96$ & 3.16 & 0.078 \\
\hline RPA & $3.22 \pm 0.62$ & $3.59 \pm 1.24$ & $3.79 \pm 1.07$ & 6.35 & $0.013^{*}$ \\
\hline AAo & $5.97 \pm 1.35$ & $7.05 \pm 1.51$ & $7.45 \pm 1.48$ & 21.41 & $<0.001^{*}$ \\
\hline \multicolumn{6}{|c|}{$\mathrm{CSA}_{\min }, \mathrm{Cm}^{2}( \pm \mathrm{SD})$} \\
\hline MPA & $5.19 \pm 1.22$ & $5.09 \pm 0.96$ & $4.82 \pm 0.90$ & 2.99 & 0.109 \\
\hline LPA & $2.71 \pm 0.62$ & $2.93 \pm 0.98$ & $3.03 \pm 0.85$ & 3.08 & 0.082 \\
\hline RPA & $2.46 \pm 0.51$ & $2.79 \pm 0.99$ & $2.95 \pm 0.80$ & 7.86 & $0.006^{*}$ \\
\hline AAo & $5.08 \pm 1.27$ & $6.28 \pm 1.39$ & $6.77 \pm 1.45$ & 31.02 & $<0.001^{*}$ \\
\hline \multicolumn{6}{|c|}{$\mathrm{CSA}_{\mathrm{avg}}, \mathrm{Cm}^{2}( \pm \mathrm{SD})$} \\
\hline MPA & $5.87 \pm 1.27$ & $5.61 \pm 1.09$ & $5.29 \pm 1.03$ & 5.23 & $0.024^{*}$ \\
\hline LPA & $3.03 \pm 0.62$ & $3.24 \pm 1.12$ & $3.37 \pm 0.91$ & 2.59 & 0.088 \\
\hline RPA & $2.88 \pm 0.57$ & $3.22 \pm 1.13$ & $3.39 \pm 0.98$ & 6.19 & $0.014^{*}$ \\
\hline AAo & $5.59 \pm 1.30$ & $6.78 \pm 1.46$ & $7.19 \pm 1.47$ & 26.00 & $<0.001^{*}$ \\
\hline \multicolumn{6}{|c|}{$\mathrm{CSA}_{\text {ratio, }}, 1( \pm \mathrm{SD})$} \\
\hline MPA/AAO & $1.05 \pm 0.23$ & $0.83 \pm 0.17$ & $0.74 \pm 0.19$ & 51.56 & $<0.001^{*}$ \\
\hline \multicolumn{6}{|c|}{$\mathrm{CSA}_{\text {change, }} \%( \pm \mathrm{SD})$} \\
\hline MPA & $20.78 \pm 5.30$ & $16.35 \pm 5.04$ & $16.17 \pm 5.03$ & 17.06 & $<0.001^{*}$ \\
\hline LPA & $18.56 \pm 5.59$ & $17.13 \pm 3.55$ & $18.29 \pm 5.29$ & 0.08 & 0.773 \\
\hline RPA & $23.69 \pm 5.52$ & $22.23 \pm 5.09$ & $21.64 \pm 6.01$ & 2.85 & 0.094 \\
\hline AAo & $15.06 \pm 5.85$ & $10.99 \pm 4.09$ & $9.26 \pm 3.32$ & 32.97 & $<0.001^{*}$ \\
\hline
\end{tabular}

CSA cross-sectional-area, SD standard deviation, *statistically significant

and right pulmonary artery and the AAo. Also, the diastolic peak flow volume was lower in older patients and overall mean flow volume $\left(\mathrm{Q}_{\text {mean }}\right)$ and, accordingly, cardiac output as measured in the ascending aorta was higher in younger participants (group $1>2>3$ ). When comparing hemodynamic parameters between the LPA and the RPA intra-individually, it occurred that peak
$\left(\mathrm{V}_{\max } ; p<0.001\right)$ and average velocities $\left(\mathrm{V}_{\text {avg }} ; p=0.001\right)$, peak $\left(\mathrm{Q}_{\text {max-sys }} ; p=0.003\right)$ and mean $\left(\mathrm{Q}_{\text {mean }} ; p<0.001\right)$ flow were higher in the RPA, while the fraction of retrograde flow was higher in the LPA $(p<0.001)$ (see Table 4).

\section{Age as an independent predictor of hemodynamic change}

After adjustment for cardiovascular risk factors (i.e., arterial hypertension and hypercholesterolemia) and bodymass-index, age was found to be an independent predictor of mean flow ( $\mathrm{Q}_{\text {mean-MPA }}(r=-0.22, p=$ $0.018), \mathrm{Q}_{\text {mean-LPA }}(r=-0.45, p<0.001), \mathrm{Q}_{\text {mean-RPA }}(r=$ $\left.-0.28, p=0.002), \mathrm{Q}_{\text {mean-AAo }}(r=-0.37, p<0.001)\right)$, maximum flow $\left(\mathrm{Q}_{\max -\mathrm{LPA}}(r=-0.30, p=0.001), \mathrm{Q}_{\max }\right.$ RPA $(r=-0.24, p=0.008))$, fraction of retrograde flow $\left(\mathrm{RF}_{\mathrm{MPA}}(r=-0.22, p=0.017), \mathrm{RF}_{\mathrm{LPA}}(r=-0.26, p=\right.$ $0.004))$, cardiac output $(r=-0.33, p<0.001)$, mean velocities $\left(\mathrm{V}_{\text {mean-AAo }}(r=-0.47, p<0.001), \mathrm{V}_{\text {mean-LPA }}(r=\right.$ $\left.-0.48, \quad p<0.001), \quad \mathrm{V}_{\text {mean-RPA }}(r=-0.45, \quad p<0.001)\right)$, maximum velocities $\left(\mathrm{V}_{\text {max-AAo }}(r=-0.59, p<0.001)\right.$, $\mathrm{V}_{\text {max-LPA }}(r=-0.39, p<0.001), \mathrm{V}_{\max -\mathrm{RPA}}(r=-0.32, p<$ $0.001))$, minimum CSA (CSA min-AAo $(r=0.35, p<$ $0.001)$ ), maximum CSA ( $\mathrm{CSA}_{\max -\mathrm{MPA}}(r=-0.25, p=$ $\left.0.005), \mathrm{CSA}_{\text {max-AAo }}(r=0.28, p=0.002)\right)$, mean CSA $\left(\mathrm{CSA}_{\text {mean-MPA }}(r=-0.22, p=0.017), \mathrm{CSA}_{\text {mean-AAo }}(r=\right.$ $0.32, p<0.001))$, MPA/AAo-CSA ratio $(r=-0.50, p<$ $0.001)$ and CSA $_{\text {change-AAo }}(r=-0.41, p<0.001)$.

\section{D visualization of flow in the main pulmonary artery}

We were able to visualize retrograde blood flow in the main pulmonary artery in all but one participant. In all cases the posterior wall of the MPA was affected by flow reversal. The mean extent of retrograde flow was $0.9-1.4 \mathrm{~cm}$ $(\mathrm{F}(2124)=0.56 ; p=0.574)$. During systole, abnormal vortices (Fig. 4 and Additional file 1: Supplemental video 1) occurred in the MPA in four male subjects with the age of 20, 34, 37, and 60. These subjects had no history of $\mathrm{PH}$ and showed no pathological findings in TTE examination.
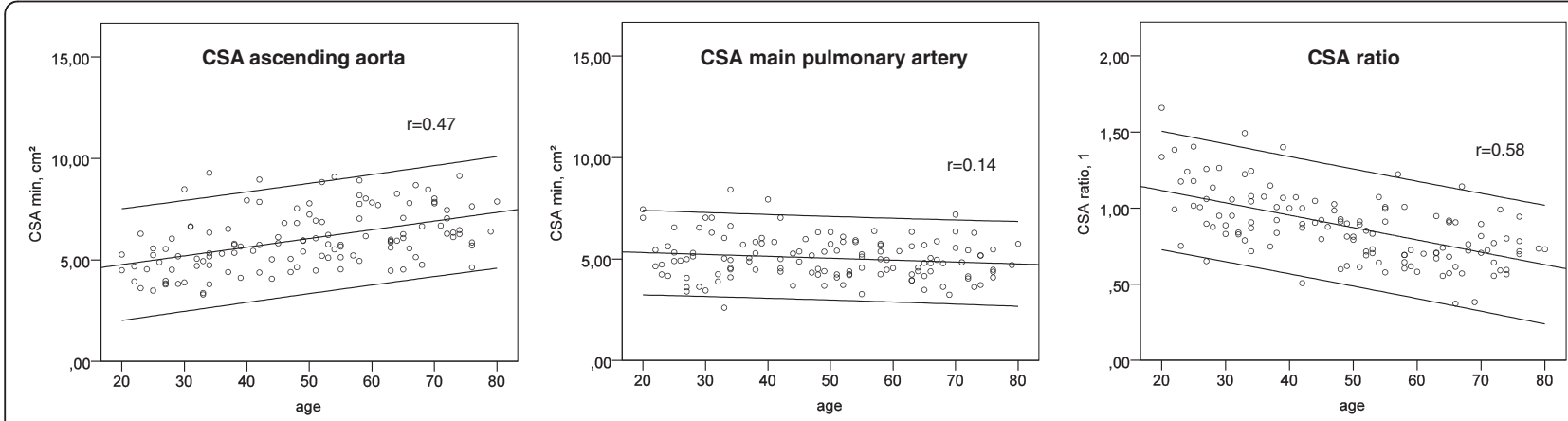

Fig. 2 Cross-sectional area of the main pulmonary artery and ascending aorta. Scatter-plots indicating the positive association between diastolic CSA in the ascending aorta and age $(r=0.47 ; p<0.001)$, the lack of an significant association between the CSA of the main pulmonary artery and age $(r=0.14 ; p=0.11)$, and the negative association between the CSA ratio and age $(r=-0.58 ; p<0.001)$; the upper and lower line are indicating the $95 \%$ confidence interval of the trend line 


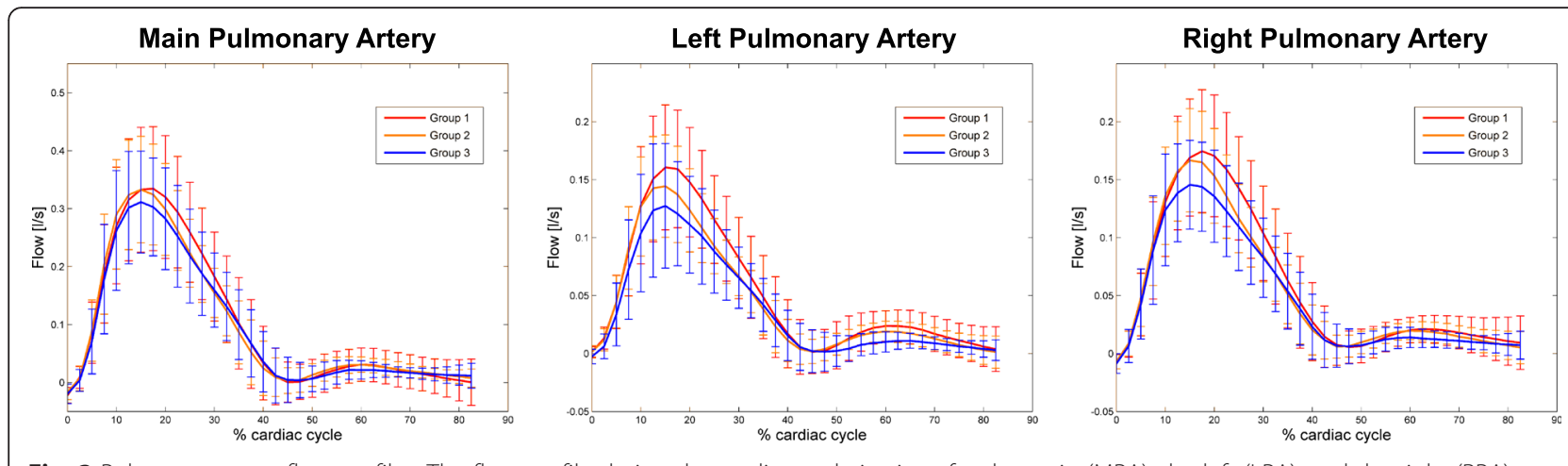

Fig. 3 Pulmonary artery flow profiles. The flow profile during the cardiac cycle is given for the main (MPA), the left (LPA), and the right (RPA) pulmonary artery. Groups are represented by different colors (red: group 1 (age 20-39); orange: group 2 (age 40-59); blue: group 3 (age 60-80))

\section{Discussion}

In this study, we performed 4D flow CMR of the pulmonary arteries in an age-stratified sample of 126 residents of Freiburg, a city in southern Germany with a population of about 230,000. To our knowledge, this is the first population-based study using 4D flow CMR to systematically analyze pulmonary hemodynamics. The purpose of our study was to provide reference values to allow interpretation of findings in future studies using 4D flow CMR, which may both be used as a diagnostic and screening tool if robust prediction of pulmonary hypertension should be possible in the future. 4D flow CMR measures proofed to be highly reliable for the quantification of planar flow in the aorta along with high

Table 3 Landmarks of the cardiac cycle

\begin{tabular}{|c|c|c|c|c|c|}
\hline & $\begin{array}{l}\text { Group } 1 \\
\text { (20-39y.) } \\
n=43\end{array}$ & $\begin{array}{l}\text { Group } 2 \\
(40-59 y .) \\
n=44\end{array}$ & $\begin{array}{l}\text { Group } 3 \\
(60-80 y .) \\
n=39\end{array}$ & $F(2,375)$ & $p$-value \\
\hline \multicolumn{6}{|c|}{$\mathrm{TTP}_{\mathrm{sys}}, \%( \pm \mathrm{SD})$} \\
\hline MPA & $17.25 \pm 3.58$ & $15.31 \pm 3.02$ & $15.38 \pm 3.20$ & 6.70 & $0.011^{*}$ \\
\hline LPA & $17.32 \pm 6.29$ & $14.65 \pm 2.46$ & $15.47 \pm 2.50$ & 3.97 & $0.049^{*}$ \\
\hline RPA & $18.23 \pm 3.52$ & $16.24 \pm 2.62$ & $16.56 \pm 7.92$ & 2.20 & 0.140 \\
\hline AAo & $15.06 \pm 4.59$ & $14.89 \pm 5.48$ & $13.46 \pm 2.70$ & 2.62 & 0.108 \\
\hline \multicolumn{6}{|c|}{$\mathrm{TTP}_{\text {ret, }} \%( \pm \mathrm{SD})$} \\
\hline MPA & $43.52 \pm 7.45$ & $43.16 \pm 5.91$ & $43.53 \pm 4.63$ & 0.00 & 0.994 \\
\hline LPA & $45.26 \pm 8.30$ & $44.52 \pm 8.29$ & $50.97 \pm 13.58$ & 6.37 & $0.013^{*}$ \\
\hline RPA & $46.69 \pm 10.01$ & $49.02 \pm 13.89$ & $49.97 \pm 13.65$ & 1.38 & 0.242 \\
\hline AAo & $41.18 \pm 9.614$ & $39.51 \pm 6.17$ & $40.6 \pm 4.33$ & 1.40 & 0.709 \\
\hline \multicolumn{6}{|c|}{$\mathrm{TTP}_{\text {dia }} \%( \pm \mathrm{SD})$} \\
\hline MPA & $59.85 \pm 9.296$ & $59.37 \pm 6.307$ & $60.53 \pm 8.112$ & 0.15 & 0.701 \\
\hline LPA & $61.06 \pm 8.991$ & $60.99 \pm 7.276$ & $66.76 \pm 10.14$ & 8.54 & $0.004^{*}$ \\
\hline RPA & $61.72 \pm 10.15$ & $62.63 \pm 9.908$ & $63.11 \pm 10.83$ & 0.38 & 0.54 \\
\hline AAo & $55.71 \pm 10.03$ & $57.76 \pm 7.792$ & $61.96 \pm 9.991$ & 9.26 & $0.003^{*}$ \\
\hline
\end{tabular}

Time-to-peak (TTP) values in \% of the cardiac cycle for peak antegrade systolic $\left(T T P_{\text {sys }}\right)$ and diastolic $\left(T \mathrm{P}_{\text {dia }}\right)$, and early diastolic retrograde flow $\left(T^{2} P_{\text {ret }}\right) . S D$ standard deviation. *statistically significant
Intra- and Inter-Observer agreement as shown previously [15]. Furthermore, 4D flow CMR flow measurements have been validated in phantom studies using predefined flow volumes and in comparison with established standard sequences [16]. In our study, the principle of conservation of mass showed excellent agreement when comparing mean flow volumes at the MPA, the combined LPA and RPA, and the AAo along with high correlations. These results indicate that our 4D flow CMR protocol can be robustly used for measuring hemodynamic parameters in the pulmonary arteries.

PA diameter is routinely used to identify $\mathrm{PH}$, as $\mathrm{PH}$ is associated with distended proximal pulmonary arteries [17]. However, the definition of PA diameter cut-off values remains problematic. Data from a previous study which examined $51 \mathrm{PH}$ patients $(44.8 \pm 15$ years of age) and 18 not age-matched normotensive controls (53.7 \pm 17 years of age) demonstrated that the CSA of the pulmonary arteries is significantly greater in relation to the aortic CSA in PH patients [6]. Our study groups of 4059 and 60-80 year-old subjects had a similar mean $\mathrm{CSA}_{\text {ratio }}$ of $0.83 \pm 0.17$ and $0.74 \pm 0.19$ when compared with the $\mathrm{CSA}_{\text {ratio }}$ of $0.87 \pm 0.17$ in normotensive controls in the study by Boerrigter et al. [6]. They suggested that a dilated PA may be useful for identifying patients with $\mathrm{PH}$. In our study none of the older patients ( $\geq 40$ years of age) had a $C S A_{\text {ratio }} \geq 1$. However, most of the younger participants without evidence for existent $\mathrm{PH}$ had a $\mathrm{CSA}_{\text {ratio }} \geq 1$, because the aortic CSA is usually smaller in young subjects and therefore the ratio is higher. Our data suggests that PA diameter remains relatively stable over time, contrary to aortic diameter which increases with age due to dilatative arteriopathy. Hence, changes of the aortic CSA have to be taken into account when identifying $\mathrm{PH}$ patients by an increase in $\mathrm{CSA}_{\text {ratio }}$. This may be reflected in the results of another study which indicated that the ratio of PA to AAo diameter was no predictor for mortality during a mean follow-up of 3 years [18]. Interestingly, we also detected an age 
Table 4 Parameters of pulmonary flow

\begin{tabular}{|c|c|c|c|c|c|}
\hline & $\begin{array}{l}\text { Group } 1 \\
\text { (20-39y.) } \\
n=43\end{array}$ & $\begin{array}{l}\text { Group } 2 \\
(40-59 y .) \\
n=44\end{array}$ & $\begin{array}{l}\text { Group } 3 \\
(60-80 y .) \\
n=39\end{array}$ & $F(2,375)$ & $p$-value \\
\hline \multicolumn{6}{|l|}{$\overline{V_{\max }, m / s( \pm S D)}$} \\
\hline MPA & $0.88 \pm 0.17$ & $0.88 \pm 0.16$ & $0.95 \pm 0.29$ & 1.86 & 0.175 \\
\hline LPA & $0.85 \pm 0.26$ & $0.70 \pm 0.16$ & $0.65 \pm 0.23$ & 17.74 & $<0.001^{*}$ \\
\hline RPA & $0.91 \pm 0.18$ & $0.79 \pm 0.15$ & $0.78 \pm 0.23$ & 9.22 & $0.003^{*}$ \\
\hline AAO & $0.71 \pm 0.16$ & $0.58 \pm 0.15$ & $0.46 \pm 0.14$ & 55.34 & $<0.001^{*}$ \\
\hline \multicolumn{6}{|l|}{$\mathrm{V}_{\mathrm{avg}}, \mathrm{m} / \mathrm{s}( \pm \mathrm{SD})$} \\
\hline MPA & $0.23 \pm 0.04$ & $0.23 \pm 0.04$ & $0.23 \pm 0.07$ & 0.68 & 0.795 \\
\hline LPA & $0.23 \pm 0.05$ & $0.19 \pm 0.03$ & $0.18 \pm 0.05$ & 27.40 & $<0.001^{*}$ \\
\hline RPA & $0.25 \pm 0.05$ & $0.23 \pm 0.03$ & $0.21 \pm 0.05$ & 21.97 & $<0.001^{*}$ \\
\hline AAo & $0.25 \pm 0.05$ & $0.23 \pm 0.04$ & $0.21 \pm 0.05$ & 22.25 & $<0.001^{*}$ \\
\hline \multicolumn{6}{|l|}{$\mathrm{Q}_{\text {max-sys, }} \mathrm{mL}( \pm \mathrm{SD})$} \\
\hline MPA & $354.2 \pm 98.7$ & $344.3 \pm 92.6$ & $328.8 \pm 93.2$ & 1.46 & 0.229 \\
\hline LPA & $170.4 \pm 50.3$ & $150.4 \pm 45.6$ & $133.5 \pm 56.9$ & 10.73 & $<0.001^{*}$ \\
\hline RPA & $186.4 \pm 48.5$ & $173.4 \pm 44.6$ & $157.3 \pm 41.2$ & 8.60 & $0.004^{*}$ \\
\hline AAo & $398.5 \pm 128.6$ & $343.1 \pm 113.6$ & $327.1 \pm 85.2$ & 8.39 & $0.004^{*}$ \\
\hline \multicolumn{6}{|l|}{$\mathrm{Q}_{\text {max-dia, }} \mathrm{mL}( \pm \mathrm{SD})$} \\
\hline MPA & $52.1 \pm 18.53$ & $38.6 \pm 15.62$ & $29.4 \pm 11.17$ & 43.97 & $<0.001^{*}$ \\
\hline LPA & $35.0 \pm 11.83$ & $22.2 \pm 9.04$ & $13.4 \pm 8.5$ & 96.55 & $<0.001^{*}$ \\
\hline RPA & $28.1 \pm 10.78$ & $20.5 \pm 9.71$ & $17.1 \pm 8.83$ & 25.48 & $<0.001^{*}$ \\
\hline AAo & $33.9 \pm 14.46$ & $29.5 \pm 10.74$ & $18.6 \pm 8.07$ & 36.26 & $<0.001^{*}$ \\
\hline \multicolumn{6}{|c|}{$\mathrm{Q}_{\text {mean, }} \mathrm{mL} / \mathrm{cycle}( \pm \mathrm{SD})$} \\
\hline MPA & $79.72 \pm 19.41$ & $74.89 \pm 17.12$ & $71.65 \pm 13.87$ & 4.59 & $0.034^{*}$ \\
\hline LPA & $39.01 \pm 8.11$ & $33.35 \pm 7.76$ & $29.58 \pm 8.04$ & 28.04 & $<0.001^{*}$ \\
\hline RPA & $43.07 \pm 11.05$ & $39.86 \pm 8.68$ & $37.04 \pm 8.06$ & 8.44 & $0.004^{*}$ \\
\hline AAO & $82.21 \pm 20.96$ & $73.39 \pm 15.64$ & $66.48 \pm 13.93$ & 17.13 & $<0.001^{*}$ \\
\hline \multicolumn{6}{|l|}{$\mathrm{RF}, \%( \pm \mathrm{SD})$} \\
\hline MPA & $3.41 \pm 2.74$ & $2.09 \pm 1.29$ & $2.43 \pm 1.76$ & 4.75 & $0.031^{*}$ \\
\hline LPA & $2.58 \pm 1.65$ & $1.48 \pm 1.28$ & $2.03 \pm 2.13$ & 2.16 & 0.144 \\
\hline RPA & $1.49 \pm 1.20$ & $0.95 \pm 0.79$ & $1.66 \pm 1.42$ & 0.45 & 0.504 \\
\hline AAO & $1.20 \pm 1.12$ & $1.34 \pm 1.05$ & $2.25 \pm 2.77$ & 7.08 & $0.009^{*}$ \\
\hline $\mathrm{CO}, \mathrm{L} / \min ( \pm \mathrm{SD})$ & $5.33 \pm 1.56$ & $4.77 \pm 0.99$ & $4.39 \pm 1.08$ & 11.90 & $<0.001^{*}$ \\
\hline
\end{tabular}

Peak $\left(\mathrm{V}_{\text {max }}\right)$ and mean $\left(\mathrm{V}_{\text {avg }}\right)$ velocities, peak systolic $\left(\mathrm{Q}_{\max -\mathrm{sys}}\right)$ and diastolic $\left(\mathrm{Q}_{\text {max-dia }}\right)$ antegrade flow volumes, mean flow volume per cardiac cycle $\left(\mathrm{Q}_{\mathrm{mean}}\right)$, fraction of retrograde flow (RF), and cardiac output (CO) as measured in the AAo. SD standard deviation. * statistically significant

dependent reduction of systolic to diastolic CSA change during the heart cycle, which is a further parameter of vascular stiffness and may be associated with increased mortality in $\mathrm{PH}$ patients $[17,19]$.

Flow in the MPA, LPA, and RPA was triphasic with antegrade flow in systole covering nearly $50 \%$ of the cardiac cycle, a regular short period of flow reversal, and diastolic antegrade flow. Like in other large elastic arteries, the elasticity of the pulmonary arteries contributes to the Windkessel effect which has a reservoir function by saving kinetic energy in systole and releasing it in diastole. The Windkessel effect becomes diminished with age as the pulmonary arteries loose compliance. This results in a reduced elasticity and distensibility in older subjects and explains that time-to-peak systolic flow is shorter compared to younger participants. The lower peak flow volume in older subjects on the other hand can be explained by the age-related reduction of (right-sided) cardiac function. In the absence of significant pulmonary valve insufficiency, retrograde flow was low in the pulmonary arteries and is mainly related to valve closure. The recoil of the pulmonary arteries in 


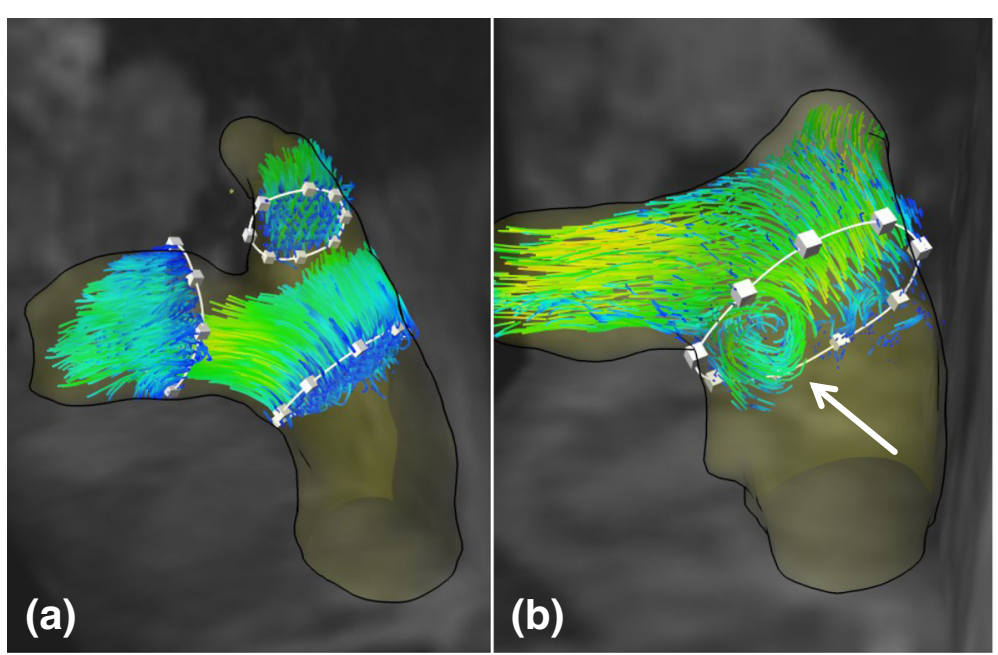

Fig. 4 Vortices in the main pulmonary artery. a A subject without appearance of vortices in the MPA. b A vortex (white arrow) appearing in the MPA during systole

diastole results in diastolic antegrade flow which, due to the loss of elasticity, was diminished in older subjects. Moreover, age-related reduction of cardiac function and vascular elasticity explains that older subjects had lower peak and mean velocities, peak systolic and diastolic antegrade flow values, and that mean flow volumes were lower in the MPA, the LPA, and the RPA when compared to younger patients. Barker et al. postulated that peak velocities, peak flow, and stroke volume were lower in $\mathrm{PH}$ patients compared to controls. We believe that part of this result may be explained by the effect of ageing, because controls $(n=19)$ were significantly younger than $\mathrm{PH}$ patients $(n=17)$ (39 vs. 57 years) in their study [5]. Furthermore, we observed that flow volumes and velocities were higher in the RPA, which can simply be explained by the inherent asymmetry of the lung and lung vascularization with three lobes on the right and two on the left side.

Vortices in the MPA during systole have previously been described to be sensitive and specific for the existence of $\mathrm{PH}$ [9]. This information is somewhat underlined by our findings with an absence of vortices of the MPA in $97 \%$ of our subjects. The occurrence of vortices in the MPA in four participants without any pathological findings in TTE may be explained by their individual geometry of the pulmonary bifurcation, which enables the formation of vortices in this area in absence of a pathologic condition. Interestingly, vortices were only detected in male subjects. However, given the low prevalence of vortices in only $3.2 \%$ of all subjects and the post-hoc nature of this finding, it requires an independent validation in a larger sample to substantiate a possible influence of geometry on vortex formation.

Potential limitations of our study were the amount of initial nonresponse to our study invitation. When compared with registry data [20] and data from another German population-based study [21] the subjects under investigation less often had hypertension, hypercholesterolemia, diabetes, were less often smokers, obese, and only few patients suffered from a cardiovascular disease. This was probably due to the recruitment modality which required participants to actively contact the study team and to visit the University Medical Center. Accordingly, particularly healthier or health-conscious residents were probably interested in collaborating in this study. Inclusion of sufficient number of young (20-29 years of age) males is a common problem in cohorts like ours. Accordingly, 14 included subjects were recruited from the personnel of our institution $(n=922$ male 20-29 years of age) on the basis of first come, first serve to minimize bias. Furthermore, the presence of comorbidity in the older age group may have influenced parameters of pulmonary flow. However, as we are interested in the dependency on age at the population level with comorbidity as a part of the natural aging process and correlations were adjusted for cardiovascular risk factors and body-mass-index, we do not believe that this has an impact on our results. Finally, due to the limited sample size we were not able to address the question of potential sex differences in the different parameters systematically. However, a potential confounding between the effects of age and sex can be excluded due to including roughly the same proportion of female subjects in each age group.

\section{Conclusions}

In conclusion, 4D flow CMR can be used to accurately and comprehensively assess flow in the pulmonary arteries in-vivo. Furthermore, we provided an overview of hemodynamic changes which occur during life in the general population independent from cardiovascular risk 
factors and body-mass. This effect of ageing should be taken into account when assessing pathologic conditions in the pulmonary arteries in future studies using 4D flow CMR to monitor effects of pulmonary hypertension.

\section{Additional file}

Additional file 1: Supplemental-video I. Occurrence of vortices in systole and retrograde flow in the main pulmonary artery in a subject without cardiovascular risk factors and no pathological findings in TTE. (MP4 $2740 \mathrm{~kb}$ )

\section{Abbreviations}

$A A o$, ascending aorta; CSA, cross-sectional-area; LPA, left pulmonary artery; MPA, main pulmonary artery; PH, Pulmonary hypertension; RF, retrograde flow; RPA, right pulmonary artery; TTP, time-to-peak

\section{Acknowledgements}

The authors thank Adriana Komancsek for conducting CMR measurements.

\section{Sources of funding}

Prof. Andreas Harloff, and Drs. Thomas Wehrum and lulius Dragonu have received funding from Deutsche Forschungsgemeinschaft (DFG), Bonn, Germany, grant \#HA5399/3-1. Prof. Constantin von zur Mühlen receives funding from Deutsche Forschungsgemeinschaft (DFG) grant \#MU2727/6-1 and \#GU1289/1-1.

\section{Authors' contributions}

Each author contributed significantly to the submitted work: TW was involved in the design of the study, performed data analysis, interpreted the data and drafted the manuscript. PH and TL recruited participants, performed CMR examinations and were involved in drafting the manuscript. W performed statistical data analyses and revised the manuscript. ID has prepared the 4D flow CMR-sequence, performed data analysis and revised the manuscript. AHe developed our 4D flow CMR-data analysis software and revised the manuscript. CvzM helped with the design of the study and revised the manuscript. JS and BN performed transthoracic echocardiograpy and revised the manuscript. AHa designed the study and revised the manuscript All authors read and approved the manuscript.

\section{Competing interests}

The authors declare that they have no competing interests.

\section{Author details}

${ }^{1}$ Department of Neurology, University Medical Center Freiburg, Breisacher Straße 64, 79106 Freiburg, Germany. ${ }^{2}$ Institute for Medical Biometry and Statistics, University of Freiburg, Freiburg, Germany. ${ }^{3}$ Department of Diagnostic Radiology - Medical Physics, University Medical Center Freiburg, Freiburg, Germany. ${ }^{4}$ Fraunhofer MEVIS, Bremen, Germany. ${ }^{5}$ Department of Cardiology, University Heart Center Freiburg, Freiburg, Germany.

Received: 16 February 2016 Accepted: 19 May 2016

\section{Published online: 31 May 2016}

\section{References}

1. Simonneau G, Robbins IM, Beghetti M, Channick RN, Delcroix M, Denton CP, Elliott CG, Gaine SP, Gladwin MT, Jing Z, Krowka MJ, Langleben D, Nakanishi N, Souza R. Updated clinical classification of pulmonary hypertension. J Am Coll Cardiol. 2009;54:543-54

2. Hoeper MM, Bogaard HJ, Condliffe R, Frantz R, Khanna D, Kurzyna M, Langleben D, Manes A, Satoh T, Torres F, Wilkins MR, Badesch DB. Definitions and diagnosis of pulmonary hypertension. J Am Coll Cardiol. 2013;62:D42-50.

3. Taleb M, Khuder S, Tinkel J, Khouri SJ. The diagnostic accuracy of Doppler echocardiography in assessment of pulmonary artery systolic pressure: a meta-analysis. Echocardiography. 2013;30:258-65.

4. Markl M, Schnell S, Barker AJ. 4D flow imaging: current status to future clinical applications. Curr Cardiol Rep. 2014;16:481.

5. Barker AJ, Roldán-Alzate A, Entezari P, Shah SJ, Chesler NC, Wieben O, Markl M, François $\mathrm{CJ}$. Four-dimensional flow assessment of pulmonary artery flow and wall shear stress in adult pulmonary arterial hypertension: results from two institutions. Magn Reson Med. 2015;73:1904-13.

6. Boerrigter B, Mauritz GJ, Marcus JT, Helderman F, Postmus PE, Westerhof N, Vonk-Noordegraaf A. Progressive dilatation of the main pulmonary artery is a characteristic of pulmonary arterial hypertension and is not related to changes in pressure. Chest. 2010;138:1395-401.

7. Sanz J, Kariisa M, Dellegrottaglie S, Prat-González S, Garcia MJ, Fuster V, Rajagopalan S. Evaluation of pulmonary artery stiffness in pulmonary hypertension with cardiac magnetic resonance. JACC Cardiovasc Imaging. 2009;2:286-95.

8. Helderman F, Mauritz G, Andringa KE, Vonk-Noordegraaf A, Marcus JT. Early onset of retrograde flow in the main pulmonary artery is a characteristic of pulmonary arterial hypertension. J Magn Reson Imaging. 2011;33:1362-8.

9. Reiter G, Reiter U, Kovacs G, Kainz B, Schmidt K, Maier R, Olschewski H, Rienmueller R. Magnetic resonance-derived 3-dimensional blood flow patterns in the main pulmonary artery as a marker of pulmonary hypertension and a measure of elevated mean pulmonary arterial pressure. Circ Cardiovasc Imaging. 2008;1:23-30.

10. Reiter U, Reiter G, Kovacs G, Stalder AF, Gulsun M a, Greiser A, Olschewski H, Fuchsjäger M. Evaluation of elevated mean pulmonary arterial pressure based on magnetic resonance $4 \mathrm{D}$ velocity mapping: comparison of visualization techniques. PLoS One. 2013:8:e82212.

11. Nogami M, Ohno Y, Koyama H, Kono A, Takenaka D, Kataoka T, Kawai H, Kawamitsu H, Onishi Y, Matsumoto K, Matsumoto S, Sugimura K. Utility of phase contrast MR imaging for assessment of pulmonary flow and pressure estimation in patients with pulmonary hypertension: comparison with right heart catheterization and echocardiography. J Magn Reson Imaging. 2009;30: 973-80.

12. Stankovic Z, Allen BD, Garcia J, Jarvis KB, Markl M. $4 D$ flow imaging with MRI. Cardiovasc Diagn Ther. 2014:4:173-92.

13. Lang RM, Bierig M, Devereux RB, Flachskampf FA, Foster E, Pellikka PA, Picard MH, Roman MJ, Seward J, Shanewise JS, Solomon SD, Spencer KT, St John Sutton M, Stewart WJ. Recommendations for chamber quantification: a report from the American Society of Echocardiography's guidelines and standards committee and the Chamber Quantification Writing Group, developed in conjunction with the European Association of Echocardiograph. J Am Soc Echocardiogr. 2005;18(12):1440-63.

14. Markl M, Harloff A, Bley TA, Zaitsev M, Jung B, Weigang E, Langer M, Hennig J, Frydrychowicz A.. Time-resolved 3D MR velocity mapping at 3T: improved navigator-gated assessment of vascular anatomy and blood flow. J Magn Reson Imaging. 2007;25:824-31.

15. Wehrum T, Kams M, Schroeder L, Drexl J, Hennemuth A, Harloff A. Accelerated analysis of three-dimensional blood flow of the thoracic aorta in stroke patients. Int J Cardiovasc Imaging. 2014;30:1571-7.

16. Frydrychowicz A, Wieben O, Niespodzany E, Reeder SB, Johnson KM, François CJ. Quantification of thoracic blood flow using volumetric magnetic resonance imaging with radial velocity encoding: in vivo validation. Invest Radiol. 2013:48:819-25.

17. Gan CT, Lankhaar JW, Westerhof N, Marcus JT, Becker A, Twisk JW, Boonstra A, Postmus PE, Vonk-Noordegraaf A. Noninvasively assessed pulmonary artery stiffness predicts mortality in pulmonary arterial hypertension. Chest. 2007;132: 1906-12.

18. Rajaram S, Swift AJ, Condliffe R, Johns C, Elliot CA, Hill C, Davies C, Hurdman J, Sabroe I, Wild JM, Kiely DG. CT features of pulmonary arterial hypertension and its major subtypes: a systematic CT evaluation of 292 patients from the ASPIRE Registry. Thorax. 2015;70:382-7.

19. Swift AJ, Rajaram S, Condliffe R, Capener D, Hurdman J, Elliot C, Kiely DG, Wild JM. Pulmonary artery relative area change detects mild elevations in pulmonary vascular resistance and predicts adverse outcome in pulmonary hypertension. Invest Radiol. 2012;47:571-7.

20. Robert Koch Institute (Publisher). Health in Germany.Federal Health Reporting. Berlin: Robert Koch Institute; 2008 http://www.rki.de/EN/Content/ Health_Monitoring/Health_Reporting/HealthlnGermany/health_in_ germany_2006/health_germany_2006_node.html. Accessed 10 May 2015.

21. Lorenz MW, Von Kegler S, Steinmetz H, Markus HS, Sitzer M. Carotid intimamedia thickening indicates a higher vascular risk across a wide age range: Prospective data from the Carotid Atherosclerosis Progression Study (CAPS). Stroke. 2006:37:87-92. 